\title{
Tweets de la autoridad sanitaria en Chile en los albores de la crisis del coronavirus
}

\section{Tweets by the health authority in Chile at the dawn of the coronavirus crisis}

Macarena Peña-y-Lilloa

a Escuela de Periodismo, Facultad de Comunicación y Letras, Universidad Diego Portales, Chile

\section{Resumen}

Introducción: En una crisis sanitaria las redes sociales son canales clave entre organismos públicos y ciudadanos. Este trabajo examina la comunicación del Ministerio de Salud de Chile en Twitter durante las semanas previas e iniciales de la crisis del Coronavirus en el país. Objetivos: Determinar si el Ministerio utilizó Twitter como un canal de comunicación directo con la ciudadanía. Métodos: Se descargaron los tweets emitidos por la cuenta oficial del Ministerio entre el 11 de febrero y el 6 de abril de 2020. Cada publicación fue clasificada según tipo (tweet, retweet, etc.); de acuerdo a si abordaba contenidos relativos al Coronavirus y si el mensaje iba dirigido a público general o a la prensa. Resultados: Los tweets emitidos por la cuenta y la proporción de éstos que referían a la pandemia aumentaron conforme avanzaban las semanas. Mientras en las semanas pre-crisis predominaron mensajes vinculados a los medios masivos, en las semanas sucesivas cobraron relevancia publicaciones dirigidas a la ciudadanía. Conclusión: La estrategia de comunicación en Twitter cambió en el transcurso de las ocho semanas analizadas probablemente debido a la extensión de los alcances de la emergencia y a modificaciones relativas a la responsabilidad organizacional en el abordaje de la crisis.

Palabras clave: Redes sociales; Comunicación de crisis; COVID-19; Comunicación de gobierno; Pandemia.

\begin{abstract}
Introduction: In a health crisis social media are key channels between public organizations and citizens. This work examines the communication of the Chilean Ministry of Health on Twitter during the previous and initial weeks of the Coronavirus crisis in the country. Goals: To determine whether the Ministry used Twitter as a direct communication channel with citizens. Methods: Tweets issued by the Ministry's official account between February 11 and April 6, 2020 were retrieved. Each post was classified by type (tweet, retweet, etc.); according to whether it addressed content related to the Coronavirus and whether the message was addressed to the general public or to the press. Results: The tweets issued by the account and the proportion of these referring to the pandemic increased as the weeks progressed. While in the pre-crisis weeks messages linked to the mass media predominated, in the weeks that followed posts directed at citizens became relevant. Conclusion: The communication strategy on Twitter changed over the course of the analyzed eight weeks, probably due to the extension of the emergency' scope and changes related to the organizational responsibility in the crisis.
\end{abstract}

Keywords: Social media; Crisis communication; COVID-19; Government communication; Pandemics. 


\section{Introducción}

as redes sociales son canales de comunicación fundamentales para las autoridades y organismos centrales de un país en contextos de crisis, pues permiten una rápida llegada tanto a los medios masivos de comunicación como a los ciudadanos (Fernández de la Hoz, 2014; Mei, Bansal, \& Pang, 2010). Esta característica es de vital importancia en crisis sanitarias como la desencadenada por la pandemia del Coronavirus en 2020, una crisis marcada por la incertidumbre en la que ciudadanos y ciudadanas están ávidos de información y en la que mensajes de fuentes oficiales compiten con mensajes de fuentes poco confiables (Depoux et al., 2020; Larson, 2018).

\section{Crisis, confianza y reputación}

El primer caso de COVID-19 en Chile se confirmó el martes 3 de marzo de 2020. Se trataba de un hombre joven que había regresado del Sudeste Asiático. A partir de ese primer reporte, todos los días sucesivos se dio cuenta de nuevos casos de personas afectadas por el virus. En el periodo que comprende el presente estudio, el país pasó de cero casos en el mes de febrero a 4.815 casos y 37 fallecidos acumulados hasta el lunes 6 de abril.

La llegada del COVID-19 a Chile interrumpió un proceso de movilizaciones sociales que se estaba desarrollando desde octubre de 2019, cuando masivas manifestaciones callejeras, motivadas en un inicio por el alza del valor del billete del transporte público y más adelante por las desigualdades económicas y sociales, pusieron en tensión al gobierno del presidente Sebastián Piñera. La pandemia y las restricciones de movimiento decretadas por el gobierno para contener el avance del virus detuvieron las manifestaciones callejeras en marzo de 2020 e incluso obligaron a posponer el plebiscito que se había acordado para el 26 de abril como parte de la salida a la crisis.

Teniendo en cuenta que la crisis sanitaria se desplegó en un contexto ya adverso para el gobierno chileno, la perspectiva con la que se estudiará la comunicación vía redes sociales en el periodo previo e inicial de la pandemia de Coronavirus en el país será la teoría situacional de la comunicación de crisis (TSCC; Coombs, 2007). De acuerdo a dicho marco conceptual, existen diversos factores que influyen en la manera en que las organizaciones abordan las crisis y cómo las manejan. Uno de esos factores es la atribución de responsabilidad, que va desde mínima hasta máxima. La responsabilidad organizacional en una crisis es mínima cuando la organización no tiene responsabilidad directa sobre ésta o cuando la crisis se origina en algo que está fuera del control de la organización. La responsabilidad es máxima, en tanto, cuando existe evidencia suficiente para señalar que la organización tiene total responsabilidad en el origen de la crisis.

El grado de responsabilidad atribuida a la organización en la crisis está a la base de los pronósticos para el daño reputacional que ésta conlleva. En esa línea, mientras un grado bajo de responsabilidad se asocia a un daño menor para la organización, una alta responsabilidad percibida se asocia a daños severos en la reputación organizacional (Coombs, 2017). Una pandemia a escala global como la del COVID-19, por tanto, cabría, al menos en sus etapas iniciales, dentro del primer tipo de responsabilidad para un ente gubernamental como el Ministerio de Salud Chileno.

Sin embargo, según la TSCC otro de los factores que inciden en cómo la crisis afecta a una organización es la reputación relacional previa, en otras palabras, cómo los stakeholders veían a la organización antes de que la crisis se desencadenara. El Estallido social chileno trajo consigo una profunda caída de la confianza ciudadana en diversas instituciones, en especial en el gobierno. Según una de las encuestas de opinión pública más reputadas en el país, la del Centro de Estudios Públicos (2020) publicada en enero de 2020 con datos de diciembre de 2019, solo un $5 \%$ de los consultados tenía confianza en el gobierno. La reputación relacional previa en el caso del gobierno chileno y en particular del Ministerio de Salud, entonces, se encontraba al inicio de la pandemia del COVID-19 en un estado no óptimo, lo que de acuerdo a la TSCC sería un factor que incidiría en el manejo comunicacional de la crisis y en sus consecuencias para la organización (Cooms, 2007).

\section{La comunicación de crisis sanitarias en las redes sociales}

Con la masificación de las redes sociales en la última década y la centralidad que tienen en las comunicaciones de las organizaciones, se vuelve fundamental observar estos canales en periodos de crisis (Eriksson, 2018). Coombs (2017) sostiene que las redes sociales permiten visibilizar la gestión comunicacional de las organizaciones en etapas de pre-crisis. Esta comunicación, que tradicionalmente se mantenía escondida del ojo público, da luces acerca de las preparaciones llevadas a cabo por los organismos para enfrentar las situaciones críticas. Por esta razón, es clave incluir las comunicaciones de este periodo en los casos de estudio de comunicación de crisis mediante redes sociales.

Diversos estudios han examinado el uso de redes sociales en contextos de emergencias sanitarias. La 
pandemia de influenza H1N1 en 2009-2010 dio origen a una serie de investigaciones acerca de cómo las organizaciones públicas y privadas se valieron de las redes sociales para comunicarse con los medios masivos y sus audiencias. Liu y Kim (2011), por ejemplo, revelaron que en dicho contexto si bien las organizaciones de salud en Estados Unidos utilizaron ampliamente las redes sociales para comunicarse directamente con sus públicos, siguieron privilegiando para este fin la comunicación a través de medios masivos. Otro estudio de las mismas autoras en dicho contexto reveló que las redes sociales fueron utilizadas por organismos de gobierno principalmente con el fin de entregar mensajes instructivos de forma directa y sin intermediarios a los ciudadanos (Kim \& Liu, 2012).

Los estudios de diversos países acerca de las comunicaciones gubernamentales mediante redes sociales en el contexto de la pandemia de 2009-2010 revelan escasa repercusión de los mensajes emitidos por cuentas de organismos públicos e importantes dificultades relativas a la credibilidad y confianza en el contexto digital. Chew y Eysenbach (2010) estudiaron el contenido de la conversación en Twitter en relación a la pandemia de influenza H1N1 y encontraron que las cuentas de las autoridades gubernamentales eran escasamente citadas en las conversaciones virtuales sobre la pandemia, a diferencia de los medios masivos, que eran importantes referentes en el debate. Un estudio de la comunicación de gobierno a través de redes sociales en Finlandia en la misma pandemia (Tirkkonen, \& Luoma-aho, 2011) mostró que la confianza de los ciudadanos en las autoridades decrecía en el entorno online en comparación con la que los ciudadanos tenían en las autoridades y su respuesta en el contexto offline. Los hallazgos de Tirkkonen y Luoma-aho (2011) muestran que a pesar de que las autoridades intentaron utilizar las redes sociales para desmentir información falsa que circulaba, carencias de oportunidad en dicha comunicación hicieron que los esfuerzos no fueran efectivos. En Chile, no existen estudios de la comunicación en redes sociales durante la pandemia de influenza H1N1 y la cuenta de Twitter del Ministerio de Salud fue creada con posterioridad a dicha emergencia sanitaria, en junio de 2010.

La crisis del Ébola en 2014, que afectó a varios países de África además de España y Estados Unidos, también dejó en evidencia deficiencias en la comunicación de las organizaciones encargadas de liderar la crisis sanitaria a través de las redes sociales. Guzmán do Nascimento (2018), en una revisión de publicaciones acerca del uso de redes sociales en la comunicación de este brote epidémico, da cuenta de una mala gestión (p. 199) de los organismos públicos, caracterizada por publicaciones con falta de inmediatez y de rigor científico, además de un desaprovechamiento de los espacios y los formatos que las redes ofrecen para comunicarse con una amplia variedad de públicos.

En el contexto de la crisis del Coronavirus en 2020 las redes sociales juegan un rol clave como medios de información de los ciudadanos, pues estos sitios y aplicaciones son mucho más masivos de lo que eran hace una década. Diversos investigadores han llamado a prestar especial atención a la circulación de información poco confiable o de plano falsa en estas redes (Depoux et al., 2020), debido a que dichas informaciones pueden generar enormes daños a la salud pública (Larson, 2018). Estudios recientemente publicados acerca de la pandemia en zonas que fueron golpeadas por el COVID-19 en los primeros meses de 2020 muestran que las redes sociales han contribuido a la difusión de información que induce al miedo entre la población, lo que se asocia a incrementos en los niveles de ansiedad en las personas (Ramazan Ahmad, Rasool, \& Gardner, 2020).

\section{Twitter como herramienta de comunicación pública}

En Chile, Twitter dista mucho de ser la red social más masiva. Estudios de compañías proveedoras de servicios de internet móvil reseñados en prensa muestran que mientras un $90 \%$ de los usuarios interactúan con Facebook, solo un 37\% lo hace con Twitter (Montes, 2018). Estos usuarios, sin embargo, son mayoritariamente personas menores de 34 años y con un alto grado de involucramiento político (Sepúlveda, 2018), un perfil similar al de quienes apoyaron más fervientemente las manifestaciones sociales que se dieron en Chile a partir de octubre de 2019 (Scherman \& Peña y Lillo, 2020). Se estima que en Chile existen alrededor de 5 millones de cuentas en Twitter, no todas ellas activas, y la cuenta del Ministerio de Salud contaba en abril de 2020 con alrededor de 375 mil seguidores.

Estudios en América Latina muestran que Twitter ha sido una herramienta regularmente utilizada por los gobiernos como un mecanismo para entregar información a los medios de comunicación y poner temas en la pauta, en especial aquellas administraciones que han preferido prescindir de instancias más directas de comunicación con los periodistas, tales como las conferencias de prensa (Amado \& Tarullo, 2015). Sin embargo, en contextos de crisis sanitarias, Twitter ha sido una herramienta escasamente utilizada por las autoridades (Calleja-Reina, Díaz Cerveró, \& Vázquez Barrio, 2017).

En Chile, el estudio del uso de Twitter como mecanismo de comunicación en la esfera pública se 
ha concentrado en la comunicación política. Un estudio de Fábrega y Paredes (2013) mostró que en la red de actores políticos chilenos en Twitter los medios de comunicación tradicionales jugaban un rol central. Los investigadores hallaron que entre los políticos era común utilizar el retweet de publicaciones de cuentas de medios de prensa como una manera de hacer broadcasting de sus dichos y actividades consignados en esos medios. En la misma línea, el estudio mostró que los diálogos políticos en la Twitósfera chilena eran inducidos en gran medida por los medios de comunicación masiva.

Teniendo en cuenta la centralidad de las redes sociales como herramientas de comunicación de los gobiernos en contextos de crisis sanitarias y el escaso conocimiento acerca del uso de Twitter para la comunicación pública de índole no política en Chile, se vuelve importante examinar la comunicación del Ministerio de Salud chileno en la red social durante las semanas previas e iniciales de la pandemia del COVID-19 en el país con el fin de determinar el rol que le dio el organismo a la comunicación directa con las audiencias mediante este canal. En síntesis, este estudio está guiado por las siguientes preguntas de investigación:

Pregunta 1: ¿En qué medida la comunicación del Ministerio de Salud en Twitter estaba orientada a la ciudadanía?

Pregunta 2: ¿Cómo cambia la comunicación del Ministerio de Salud en Twitter desde la etapa previa a la crisis a las etapas siguientes en la que ésta ya se ha manifestado?

\section{Objetivos}

En este artículo se analiza el uso de la red social Twitter por parte del Ministerio de Salud chileno entre febrero y abril de 2020, periodo que abarca tres semanas antes de que la crisis se desencadenara con el primer caso de COVID-19 en el país y las cinco semanas siguientes a aquella primera notificación. El propósito de este estudio es determinar el grado en el cual la autoridad sanitaria chilena utilizó durante el periodo la red social Twitter como un canal de comunicación directo con la ciudadanía, considerando que esta crisis sanitaria llegó a Chile en medio de una crisis social y política y ad portas de un plebiscito para la elaboración de una nueva Constitución (Aste Leiva, 2020).

\section{Método}

Para caracterizar la estrategia comunicacional utilizada en la red social Twitter por el Ministerio de Salud de Chile durante las primeras semanas de la crisis sanitaria por el COVID-19 en el país, se descargaron los tweets de la cuenta del Ministerio de Salud (@ministeriosalud) emitidos durante febrero, marzo y parte de abril de 2020, usando el software NodeXL Pro y su herramienta "Importar la red de un usuario de Twitter". NodeXL Pro es un template de Excel para el análisis de redes sociales y entre sus funcionalidades permite descargar publicaciones de Twitter y otras redes sociales especificando un tema, hashtag, o nombre de usuario. Para este estudio se descargó la red básica del usuario @ministeriosalud, que muestra los tweets originales, las respuestas, menciones y retweets de dicha cuenta.

Para decidir el margen temporal de los tweets que se analizarían, se tomó como eje el día martes 3 de marzo, que es la fecha de la notificación del primer caso de COVID-19 en el país. Con el fin de capturar actividad en la red social anterior a dicho hito, se consideraron tweets emitidos las tres semanas previas a la fecha eje, es decir, entre el martes 11 de febrero y el lunes 2 de marzo (semanas 1 a 3). Además, y con el fin de dar cuenta de las primeras semanas de la crisis, se seleccionaron los tweets emitidos en las cinco semanas posteriores, es decir, entre el martes 3 de marzo y el lunes 6 de abril (semanas 4 a 8). En total, se descargaron 4.035 tweets emitidos por @ministeriosalud durante esas ocho semanas. Sin embargo, se advirtió la presencia de tweets duplicados, pues en los casos en que el mismo tweet mencionaba a más de una cuenta, NodeXL Pro contaba a la publicación tantas veces como cuentas referidas, por lo que se realizó una depuración de la base de datos con el fin de eliminar los duplicados. Una vez realizado dicho proceso la muestra total estuvo compuesta por 2.848 tweets emitidos en el periodo estudiado (11 de febrero al 2 de abril) por la cuenta @ministeriosalud. La unidad de análisis en este estudio fue el tweet.

\section{Variables}

Las variables que se examinaron para cada uno de los tweets analizados fueron: la semana en la que fue emitido el tweet, el tipo de tweet, la presencia o ausencia de contenido relativo a la pandemia de COVID-19 y la naturaleza del mensaje contenido en el tweet, específicamente si se trataba de un mensaje dirigido a la prensa o uno dirigido a la ciudadanía.

\section{Semana}

La semana en la que fue emitido el tweet se codificó utilizando la información de fecha y hora de emisión del tweet que se obtuvo al descargar la base de datos mediante NodeXL Pro. 


\section{Tipo de Tweet}

La variable tipo de tweet se refiere a si se trataba de un tweet original, un retweet (citar un tweet de otra cuenta), una respuesta a una cuenta, o una mención a otra cuenta. Si bien esta categorización la entregaba NodeXL Pro al momento de descargar los tweets, fue necesario refinarla, pues al revisar el contenido de los tweets se pudo determinar que algunos que estaban clasificados como respuestas en realidad correspondían a menciones $(n=6)$.

\section{Contenido COVID}

Se codificó la presencia o ausencia de contenido relativo a la pandemia de COVID-19 en cada uno de los tweets. Para realizar esa clasificación, primero se examinaron los hashtags presentes en el tweet y se separó aquellos que tenían referencia a conceptos como "Coronavirus", "COVID-19" o "COVID". Los tweets que no tenían esos hashtags fueron revisados detenidamente en busca de los mismos conceptos en sus textos, además de otras ideas clave como "alerta sanitaria", "aduana sanitaria", "cordón sanitario" o "cuarentena". Un 10\% de la muestra $(n=285)$ fue analizada por un segundo codificador, quien coincidió en un $100 \%$ con la codificación original de esta variable $(\mathrm{K}=1)$.

\section{Naturaleza del mensaje}

Finalmente, y solo para aquellos tweets con contenido relativo a la pandemia de COVID-19, se codificó la naturaleza del mensaje, que buscaba separar aquellos tweets que daban cuenta de apariciones en medios masivos 0 acciones dirigidas a la prensa de aquellos tweets en los que el Ministerio entregaba información directa a la ciudadanía. Los mensajes categorizados en el primer grupo tenían al menos una de las siguientes características: mencionaban la cuenta de algún medio de comunicación, programa o periodista; presentaban citas textuales de declaraciones de algún personero ligado al Ministerio; o daban cuenta de una actividad pasada 0 futura a la que tradicionalmente es convocada la prensa (ej. inauguración de un nuevo recinto hospitalario). Los mensajes categorizados en el segundo grupo se caracterizaban por usar un lenguaje dirigido a los ciudadanos invitándolos a realizar una acción específica (ej. conductas preventivas); por entregar información concreta acerca de las medidas adoptadas (ej. zonas que entran 0 salen de cuarentenas); o por entregar información de interés general, como el caso del reporte diario de nuevos contagios, fallecidos y disponibilidad de recursos médicos y asistenciales. Un 10\% de la muestra
( $n=210)$ fue analizada en esta variable por un segundo codificador y se obtuvo un indicador fuerte $(\mathrm{K}=.82)$ de fiabilidad entre codificadores (McHugh, 2012).

\section{Resultados}

Las estadísticas descriptivas para cada una de las variables examinadas se presentan en la Tabla 1. Se observa que la mayoría de los tweets del periodo estudiado $(38,1 \%)$ corresponden a menciones, principalmente a las cuentas de las autoridades del Ministerio, como el ministro Jaime Mañalich (@ jmanalich; $n=218$ ); la subsecretaria de Salud Pública, Paula Daza (@pdazan; n = 196), o el subsecretario de Redes Asistenciales, Arturo Zúñiga (@arturozunigaj; $n=156)$. Estas menciones por lo general dan cuenta de apariciones públicas de estas autoridades y se caracterizan por reseñar sus dichos, principalmente mediante citas textuales. Más de un tercio de las publicaciones corresponden a retweets $(34,6 \%)$, principalmente de publicaciones de las autoridades del Ministerio en sus cuentas personales 0 de las cuentas de medios de comunicación que publican apariciones de las autoridades del Ministerio en dichos medios. Un $27 \%$ de las publicaciones son tweets originales y solo un $0,3 \%$ corresponde a respuestas a cuentas de otros usuarios (tabla 1).

La gran mayoría de los tweets del periodo estudiado abordan la pandemia de COVID-19 o aspectos asociados a ella $(73,3 \%)$; de ellos, un porcentaje mayoritario $(56,7 \%)$ son mensajes que dan cuenta de apariciones en medios de comunicación masiva 0 actividades diseñadas para la prensa, mientras que un $43,3 \%$ de los tweets con contenido sobre la pandemia corresponden a mensajes orientados a comunicarse directamente con la ciudadanía, ya sea entregando información acerca de la pandemia en el país, por ejemplo, los números de casos diarios o las zonas que entraban o salían de cuarentenas ( $n=$ 689; 33,0\% de los tweets sobre COVID-19) o mensajes de campañas promoviendo conductas preventivas $(n=$ $179 ; 8,6 \%$ de los tweets sobre COVID-19) tales como el lavado de manos, el uso de mascarillas, respetar la cuarentena, o llamados a buscar más información en los canales oficiales del Ministerio. La Tabla 2 presenta una muestra de los tweets clasificados en cada una de estas categorías. Mientras la gran mayoría de las publicaciones de información acerca de la pandemia dirigidas a la prensa o con apariciones de prensa fueron menciones $(62,3 \%)$ o retweets $(32,0 \%)$, las publicaciones directas para el público fueron principalmente tweets originales $(48,4 \%)$ o retweets $(38,1 \%)$ (tabla 2$)$. 
Tabla 1. Estadísticas descriptivas de la muestra.

\begin{tabular}{|c|c|c|}
\hline & Frecuencia & $\%$ del total de Tweets \\
\hline \multicolumn{3}{|l|}{ Semana } \\
\hline Tweets semana 1 (11 - 17 Febrero) & 196 & $6.9 \%$ \\
\hline Tweets semana 2 (18 - 24 Febrero) & 323 & $11.3 \%$ \\
\hline Tweets semana 3 (25 Febrero - 2 Marzo) & 354 & $12.4 \%$ \\
\hline Tweets semana 4 (3 - 9 Marzo) * primer caso & 305 & $10.7 \%$ \\
\hline Tweets semana 5 (10 - 16 Marzo) & 381 & $13.4 \%$ \\
\hline Tweets semana 6 (17 - 23 Marzo) & 459 & $16.1 \%$ \\
\hline Tweets semana 7 (24 - 30 Marzo) & 421 & $14.8 \%$ \\
\hline Tweets semana 8 (31 Marzo - 6 Abril) & 409 & $14.4 \%$ \\
\hline \multicolumn{3}{|l|}{ Tipo de Tweet } \\
\hline Tweet original & 768 & $27.0 \%$ \\
\hline Re-Tweet & 985 & $34.6 \%$ \\
\hline Respuesta & 9 & $0.3 \%$ \\
\hline Mención & 1.086 & $38.1 \%$ \\
\hline \multicolumn{3}{|l|}{ Contenido } \\
\hline Tweet con contenido COVID-19 & 2.089 & $73.3 \%$ \\
\hline \multicolumn{3}{|l|}{ Naturaleza del mensaje } \\
\hline $\begin{array}{l}\text { Tweet con contenido COVID-19 con } \\
\text { información de prensa }\end{array}$ & 1.221 & $42.9 \%$ \\
\hline $\begin{array}{l}\text { Tweet con contenido COVID-19 con } \\
\text { información para ciudadanía }\end{array}$ & 905 & $31.8 \%$ \\
\hline Total Tweets del periodo & 2.848 & $100.0 \%$ \\
\hline
\end{tabular}


Tabla 2. Ejemplos de Tweets sobre pandemia de Coronavirus por categoría.

\begin{tabular}{|c|c|c|}
\hline Categoría & Sub-categoría & Tweet \\
\hline \multirow[t]{2}{*}{$\begin{array}{l}\text { Tweet con contenido COVID-19 } \\
\text { con información de prensa }\end{array}$} & Apariciones en medios & $\begin{array}{l}\text { Subsecretaria @pdazan en @CNNChile } \\
\text { por \#PlanCoronavirus: "la etapa } 3 \text { es } \\
\text { cuando no se logra la trazabilidad, la } \\
\text { etapa } 4 \text { es cuando el brote ya está en todo } \\
\text { el país y ahí es cuando hay que hacer la } \\
\text { mitigación en los centros de atención de } \\
\text { salud". <enlace> }\end{array}$ \\
\hline & Pautas de prensa & $\begin{array}{l}\text { Subsec de Redes Asistenciales @ } \\
\text { arturozunigaj encabeza reunión con } \\
\text { representantes de prestadores privados } \\
\text { para informar sobre las directrices del } \\
\text { Plan de la Red Integrada por \#COVID_19 } \\
\text { <enlace> }\end{array}$ \\
\hline \multirow[t]{2}{*}{$\begin{array}{l}\text { Tweet con contenido COVID-19 } \\
\text { con información para ciudadanía }\end{array}$} & Información general & $\begin{array}{l}\text { [MEDIDAS] Se indicará cuarentena } \\
\text { obligatoria a todas las personas que } \\
\text { ingresen a Chile y que provengan de } \\
\text { cualquier país (residentes chilenos o } \\
\text { extranjeros). El detalle en <enlace> } \\
\text { \#CuidémonosEntreTodos }\end{array}$ \\
\hline & Campañas & $\begin{array}{l}\text { \#CuidémonosEntreTodos del Coronavirus } \\
\text { Lávate las manos frecuentemente con } \\
\text { agua y jabón. Más consejos de prevención, } \\
\text { en <enlace> }\end{array}$ \\
\hline
\end{tabular}


En la Figura 1 se muestra la tendencia de publicaciones en las ocho semanas consideradas en el análisis, entre el 11 de febrero y el 6 de abril. Como se puede ver, la cantidad de tweets emitidos por la cuenta @ministeriosalud fue creciendo conforme avanzaban las semanas, lo mismo respecto a la proporción de tweets referidos a la pandemia de COVID-19. El salto en esta proporción más visible se advierte entre la semana tres y cuatro, que es cuando se reporta el primer caso de COVID-19 en el país. Se observa además que en las primeras semanas del periodo estudiado, cuando no existían casos de COVID-19 en Chile, gran parte de las publicaciones sobre la crisis sanitaria en la red social del Ministerio correspondían a apariciones mediáticas de las autoridades 0 a pautas de prensa sobre el tema, mientras que cuando la epidemia se manifestó en el país, dichas publicaciones comenzaron a ceder espacio a aquellas con contenido especialmente dirigido a los ciudadanos, tales como mensajes promoviendo conductas preventivas 0 información práctica. Si bien entre la semana 7 y la semana 8 se vuelve a ver un alza de los contenidos asociados a pautas de prensa, las proporciones aún no se acercan a lo visto en las semanas previas al inicio de la epidemia en el país.

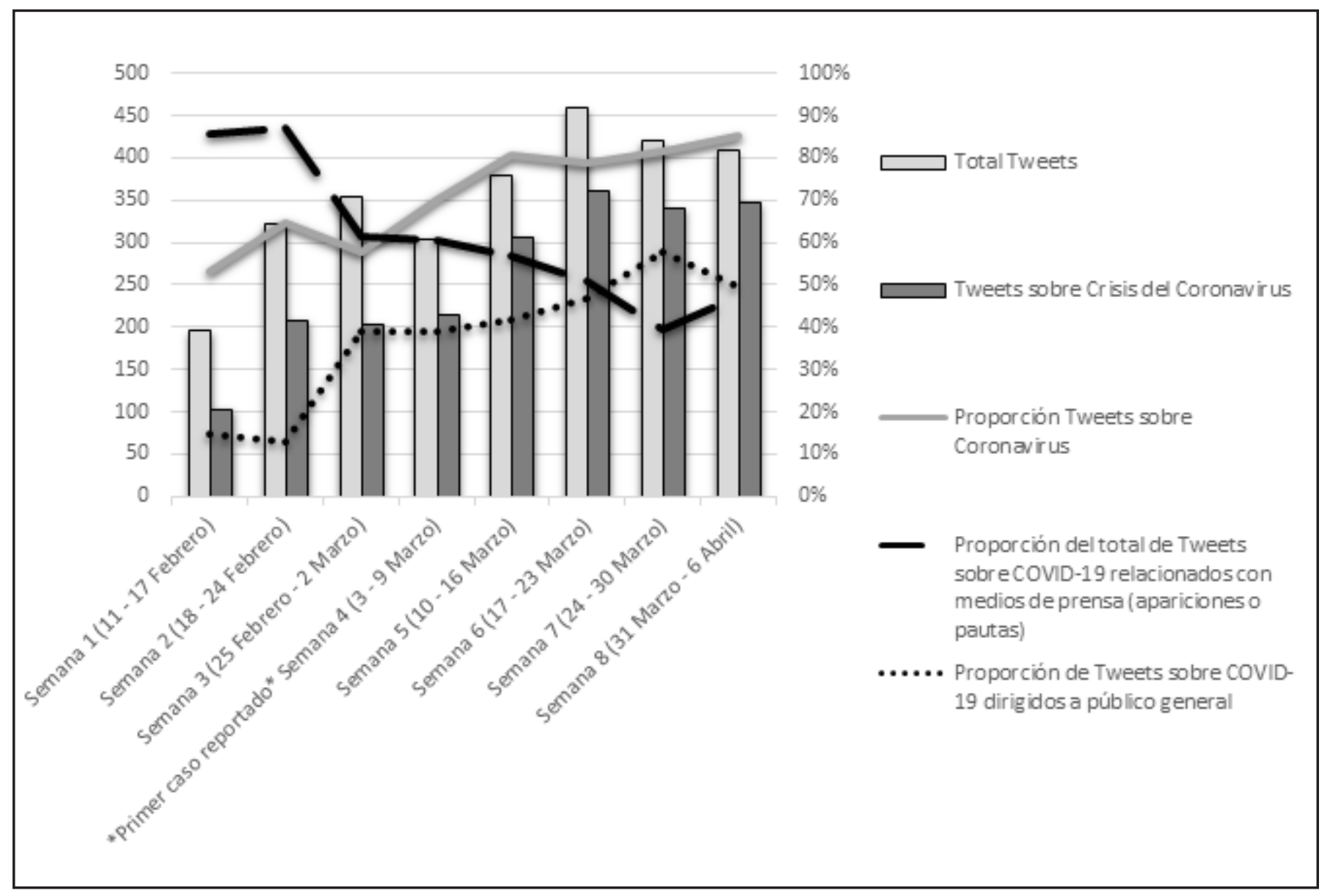

Figura 1. Tendencia de tweets publicados en las ocho semanas de análisis. Elaboración propia. 


\section{Discusión}

El presente estudio buscaba caracterizar el uso de la red social Twitter por parte del Ministerio de Salud de Chile durante el periodo previo y las semanas iniciales de la crisis del COVID-19 en el país, con el fin de determinar el grado en el cual la autoridad sanitaria utilizó este canal para llegar directamente a los ciudadanos con mensajes acerca de la pandemia o recomendaciones para enfrentarla.

Como era esperable, el número de tweets emitidos por la cuenta del Ministerio de Salud aumentó conforme avanzaban las semanas y se hacía más patente y cercana la crisis del COVID-19 en Chile (Chew \& Eysenbach, 2010). Dicha alza se puede explicar por una creciente necesidad de la autoridad sanitaria por comunicar al público acerca de la pandemia, pero también por un aumento del interés de los medios masivos de comunicación en el tema. Esta última hipótesis se vuelve plausible si se observa que la mayoría de los tweets sobre COVID-19 emitidos en el periodo previo al primer caso confirmado en Chile correspondían a retransmisiones de tweets publicados en medios de comunicación o a pautas dirigidas a la prensa. Es importante señalar, sin embargo, que el menor número de tweets del Ministerio en las semanas correspondientes al mes de febrero puede deberse a que ese mes es tradicionalmente periodo de vacaciones en el país, mientras que marzo es el mes en que se reinician con normalidad todas las actividades, por ejemplo, las clases en escuelas y universidades.

Los tweets de la autoridad sanitaria revelan que en las semanas previas a la confirmación del primer caso de COVID-19 en Chile los esfuerzos se concentraron en mostrar los preparativos que se estaban realizando para la inminente llegada del virus al país. Una mirada casuística al contenido de los tweets en ese periodo revela un énfasis en presentar simulacros de reacción ante posibles contagios en hospitales a lo largo del país, inauguraciones de recintos asistenciales 0 ampliaciones de éstos, y declaraciones en medios masivos dando cuenta de los recursos médicos con los que el país contaba para enfrentar la crisis. En ese sentido, el comportamiento comunicacional del Ministerio en Twitter es consistente con lo previsto por Coombs (2017) respecto de la publicidad de la etapa precrisis en las redes sociales de las organizaciones. No obstante, llama la atención que la mayoría de dichas publicaciones no corresponden a mensajes dirigidos especialmente a las audiencias sino que orientados a los medios masivos. La centralidad de los medios masivos de comunicación en la discusión acerca de crisis sanitarias en redes sociales ha sido advertida en estudios previos en otros brotes epidémicos, como en el caso del Ébola en España (Percastre-Mendizábal, PontSorribes, \& Suau-Gomila, 2019).

Consistente con los hallazgos de investigaciones realizadas en la pandemia de influenza H1N1 en 20092010 (Kim \& Liu, 2012; Tirkkonen, \& Luoma-aho, 2011), cuando la crisis del COVID-19 ya se hizo manifiesta en Chile la autoridad sanitaria comenzó a utilizar con más fuerza su cuenta de Twitter para transmitir mensajes educativos promoviendo comportamientos preventivos y para entregar instrucción e información práctica respecto de cómo abordar la crisis. Si bien estas acciones pueden resultar valiosas para promover comportamientos que ayuden en el control de la pandemia, para ser verdaderamente efectivas y sobre todo, para contrarrestar los efectos negativos de noticias falsas y teorías conspirativas en las redes, es importante anticiparse a las crisis y actuar proactivamente estableciendo autoridad sobre la información y las versiones oficiales (Jin, Liu, \& Austin, 2011).

Si bien desde la perspectiva de la TSCC (Coombs, 2007) una pandemia puede ser vista como una crisis de responsabilidad mínima para un organismo como el Ministerio de Salud de Chile, cuando la crisis se materializa en el país y comienza a avanzar, el gobierno es obligado a rendir cuentas acerca de las decisiones adoptadas para contenerla. En ese sentido, la responsabilidad percibida puede aumentar, considerando que de las medidas gubernamentales depende el devenir de la crisis en el país. Es posible que el giro en la estrategia de comunicación en la red social Twitter del Ministerio desde una comunicación más bien reactiva a una más proactiva se deba, precisamente, a que cuando comenzaron a aumentar los casos de COVID-19 en el país el organismo se apropió de la responsabilidad y decidió no solo aumentar el volumen de publicaciones, sino que también dirigirlas con más claridad a los ciudadanos sin pasar por los medios masivos como intermediarios. Esta comunicación directa desde el organismo es fundamental considerando el contexto de desconfianza en el que se inició la crisis sanitaria y a que la gestión de la autoridad de salud fue uno de los blancos más consistentes de las protestas iniciadas en octubre de 2019, principalmente debido al acceso limitado o de mala calidad a servicios de salud y la profunda desigualdad en salud que existe en Chile (Castillo-Laborde et al., 2017).

Este trabajo tiene al menos dos limitaciones. La primera es que este estudio se refiere a un periodo estrecho de tiempo en el cual la crisis no se ha desplegado 
en plenitud, pues al cierre de este trabajo la curva de contagios de COVID-19 en Chile seguía en ascenso y el número de fallecidos iba también al alza. Sería relevante examinar las comunicaciones del Ministerio mediante la red social Twitter a lo largo de toda la crisis sanitaria con el fin de confirmar si es que las tendencias encontradas en el presente estudio se mantienen o se modifican. Una segunda limitación es que a diferencia de estudios desarrollados en el contexto de la pandemia de influenza H1N1 o del Ébola y que dan cuenta de la discusión acerca de estos tópicos en las redes en un determinado momento (ej. Chew \& Eysenbach, 2010; Guidry, Jin, Orr, Messner, \& Meganck, 2017), este estudio se centra en los tweets emitidos solo por el usuario del Ministerio de Salud en la red social y por ende no da cuenta cabalmente acerca de debates o deliberaciones que pudiesen estar teniendo lugar en la red y en las que dicho usuario no intervenga. Futuros estudios de esta pandemia se beneficiarían de una perspectiva de red para examinar tendencias no solo en la emisión de contenidos, sino en las conversaciones que tuvieron lugar durante la crisis.

\section{Conclusiones}

Esta investigación aporta una mirada a las tendencias en la comunicación del Ministerio de Salud en las semanas previas e iniciales de la pandemia del COVID-19 en Chile. Se observa un aumento del volumen de publicaciones en la cuenta y de publicaciones asociadas a la pandemia conforme avanza el tiempo y se extiende la crisis en el país, a la vez, se observa un incremento en la proporción de mensajes dirigidos a los y las ciudadanas en desmedro de los mensajes que reseñan contenidos de prensa 0 contenidos orientados a periodistas. Este cambio de estrategia de la comunicación en Twitter puede ser atribuible a la extensión de los alcances de la emergencia y a modificaciones relativas a la responsabilidad organizacional en su abordaje.

Este trabajo tiene además implicaciones prácticas, puesto que puede ser considerado el punto de partida de una evaluación de la gestión comunicacional de un organismo público en medio de una crisis sanitaria. En esa línea, podría ser un insumo para el diseño de planes de comunicación para emergencias y crisis sanitarias que den más centralidad a las redes sociales como canal de comunicación directo con la ciudadanía.

\section{Referencias bibliográficas}

Amado, A., \& Tarullo, R. (2015). Tuitear para agendar: el uso de Twitter como gacetilla de prensa en la comunicación gubernamental. Revista Mexicana de Opinión Pública, 19, 127-145. https://doi. org/10.1016/j.rmop.2015.02.003

Aste Leiva, B. (2020). Estallido social en Chile: la persistencia de la Constitución neoliberal como problema. DPCE Online, 42(1), 3-19. Recuperado de: http://www.dpceonline.it/index.php/dpceonline/ article/view/885

Calleja-Reina, M.A., Díaz Cerveró, E., \& Vázquez Barrio, T. (2017). Dengue en Corrientes (Argentina): Estrategia de comunicación en Twitter durante el brote de 2016. Revista Española de Comunicación en Salud, 8(2), 118-134, doi:10.20318/recs.2017.3996

Castillo-Laborde, C., Aguilera-Sanhueza, X., HirmasAdauy, M., Matute, W. I., Delgado-Becerra, I., Nájera-De Ferrari, M., ... González-Wiedmaier C. (2017). Health insurance scheme performance and effects on health and health inequalities in Chile. MEDICC Review, 19, 57-64. Recuperado de: https://www.medigraphic.com/cgi-bin/new/resumen. cgi?IDARTICULO=74082

Centro de Estudios Públicos. (2020, enero). Estudio Nacional de Opinión Pública $N^{\circ} 84$, Diciembre 2019. Recuperado de: https://www.cepchile.cl/ cep/encuestas-cep/encuestas-2009-2018/estudionacional-de-opinion-publica-n-84-diciembre-2019

Chew, C., \& Eysenbach, G. (2010). Pandemics in the age of Twitter: Content analysis of Tweets during the 2009 H1N1 Outbreak. PloS One, 5(11), 1-13. https://doi.org/10.1371/journal.pone.0014118

Coombs, W. T. (2007). Protecting organization reputations during a crisis: The development and application of situational crisis communication theory. Corporate Reputation Review, 10(3), 163-176. doi:10.1057/ palgrave.crr.1550049

Coombs, W. T. (2017). Revising situational crisis communication theory: The influences of social media on crisis communication theory and practice. In L. Austin \& Y. Jin (Eds.) Social Media and Crisis Communication (pp. 41-58). New York, EUA: Routledge.

Depoux, A., Martin, S., Karafillakis, E., Bsd, R. P., WilderSmith, A., \& Larson, H. (2020). The pandemic of social media panic travels faster than the COVID-19 Outbreak. Journal of Travel Medicine. Recuperado de: 10.1093/jtm/taaa031 
Eriksson, M. (2018). Lessons for crisis communication on social media: A systematic review of what research tells the practice. International Journal of Strategic Communication, 12(5), 526-551. doi:10.1080/1553 118X.2018.1510405

Fábrega, J., \& Paredes, P. (2013). La política chilena en 140 caracteres. In A. Arriagada \& Navia, P. (Eds.) Intermedios. Medios de Comunicación y Democracia en Chile (pp. 201-225). Santiago, Chile: Ediciones Universidad Diego Portales.

Fernández de la Hoz, K. (2014). La comunicación en crisis sanitarias: perspectiva de las administraciones públicas. Revista Española de Comunicación en Salud, 5(1), 14-19. Recuperado de: https://erevistas.uc3m.es/index.php/RECS/article/view/3349

Guidry, J. P. D., Jin, Y., Orr, C. A., Messner, M., \& Meganck, S. (2017). Ebola on Instagram and Twitter: How health organizations address the health crisis in their social media engagement. Public Relations Review, 43(3), 477-486. doi:https://doi. org/10.1016/j.pubrev.2017.04.009

Guzmán do Nascimento, B. (2018). Comunicación y salud: La gestión de la crisis del Ébola a través de las redes sociales. Revista Española de Comunicación en Salud, 9(2), 196-202. doi:10.20318/recs.2018.4497

Jin, Y., Liu, B. F., \& Austin, L. L. (2011). Examining the role of social media in effective crisis management: The effects of crisis origin, information form, and source on publics' crisis responses. Communication Research, 41(1), 74-94. doi:10.1177/0093650211423918

Kim, S., \& Liu, B. F. (2012). Are all crises opportunities? A comparison of how corporate and government organizations responded to the 2009 flu pandemic. Journal of Public Relations Research, 24(1), 6985. doi:10.1080/1062726X.2012.626136

Larson, H. J. (2018). The biggest pandemic risk? Viral misinformation. Nature, 562, 309. doi: 10.1038/ d41586-018-07034-4

Lim, R. S-q, Tan, E. Y., Aziz, N. B. A., \& Pang, A. (2018). When a pandemic strikes. Toward the social media pandemic communication model. In L. Austin \& Y. Jin (Eds.) Social Media and Crisis Communication (pp. 253-266). New York, EUA: Routledge.

Liu, B. F., \& Kim, S. (2011). How organizations framed the
2009 H1N1 pandemic via social and traditional media: Implications for U.S. health communicators. Public Relations Review, 37(3), 233-244. doi:https://doi. org/10.1016/j.pubrev.2011.03.005

McHugh, M. L. (2012). Interrater reliability: the kappa statistic. Biochemia Medica, 22(3), 276-282. Recuperado de https://pubmed.ncbi.nlm.nih. gov/23092060

Mei, J. S. A., Bansal, N., \& Pang, A. (2010). New media: a new medium in escalating crises? Corporate Communications: An International Journal, 15(2), 143-155. doi:10.1108/13563281011037919

Montes. C. (2018, Marzo). Diez años de Facebook en Chile. Recuperado de: https://www.latercera. com/tendencias/noticia/diez-anos-Facebookchile/106698/

Percastre-Mendizábal, S., Pont-Sorribes, C., \& SuauGomila, G. (2019). La gestión comunicativa en redes sociales digitales de la emergencia del Ébola en España. Revista Española de Comunicación en Salud, Suplemento(1), S89-S90. doi:10.20318/ recs.2019.4437

Ramazan Ahmad, A., Rasool, H., \& Gardner, M. (2020). The impact of social media on hyped panic during the COVID-19 pandemic: The Iraqi Kurdistan case. Journal of Medical Internet Research Mental Health. in print. doi:10.2196/19556

Sepúlveda, P. (2018, septiembre) ¿Quiénes son los usuarios de Twitter en Chile? Recuperado de: https://www.latercera.com/tendencias/noticia/ quienes-los-usuarios-Twitter-chile/304944/

Scherman, A. \& Peña-y-Lillo, M. (2020, enero). Se instala un mayor respaldo de los jóvenes a la violencia en la acción política. Recuperado de: https://ciperchile.cl/2020/01/28/se-instala-un-mayorrespaldo-de-los-jovenes-a-la-violencia-en-la-accionpolitical

Tirkkonen, P., \& Luoma-aho, V. (2011). Online authority communication during an epidemic: A Finnish example. Public Relations Review, 37(2), 172174. doi:10.1016/j.pubrev.2011.01.004 